\title{
Application of RFID-based Context Awareness in Mobile Learning
}

\author{
Yu Lei ${ }^{1, a^{*}}$, Guo Yong ${ }^{1, b}$ \\ ${ }^{1}$ College of Computer Science \& Engineering, Xi'an University of Technology, Xi'an, China \\ ayulei@xaut.edu.cn, ${ }^{\mathrm{b}}$ guoyong@stu.xaut.edu.cn
}

Keywords: RFID; M-learning; Context Awareness

Abstract. If learning objects distributed in the environment can directly interact with mobile equipment of the learners through environmental awareness technology, the most appropriate learning content can be presented, learners can stay in mobile learning stably, and original learning effect can not be affected due to obtaining unsuitable learning content in order to achieve close situation interaction between mobile learning and real learning environment. Therefore, environmental awareness technology is applied in the mobile learning, and mobile learning can be converted into ubiquitous learning. In the paper, RFID is adopted for constructing context awareness model aiming at application of mobile learning in libraries, museums, zoos, historical sites, botanical garden and other real learning environments, and learning recommendation algorithm under the model is given.

\section{Introduction}

Integration of computer into environment or daily tool is emphasized in context awareness computing. Computer itself can disappear from people's line of sight, and people's focus center can return to the to-be-completed task. It integrates techniques in many aspects of computing, communications, mobile devices, sensor technology, software, etc. Computing concept integrated with computing environment is stressed. Currently, various applications with intelligent mobile phone as carrier are explosively developed, wherein mobile learning, as an emerging learning mode is especially popular among young people.

In foreign countries, Ericsson Education, Norway NKI Distance Education Institutions, Open University in Germany, Ireland International Distance Education Institutions and Rome No. 3 Open University cooperate and develop the product, which is regarded as 'pioneer of learning in the future'. The project aims at designing a virtual learning environment for mobile technology. Learning environment model is proposed [1]. A mobile learning system of learning language with fragmented time is developed by learning experiment in Stanford University. Surrounding environment is perceived by mobile equipment for the first time, thereby selecting learning contents for learners automatically [2]. In China, 'mobile teaching' projects of Education Ministry are also carried out in recent years[3], such as mobile teaching network system cooperated by Nanjing University and Japan Panasonic Mobile Communication, etc. However, the key issue of integrating environment into learning is ignored in all projects.

\section{RFID-based Context Modeling}

There are many approaches to obtain context information. Information sources include sensors, RFID read-write device, cameras, etc. Next, context modeling aims at establishing unified abstract logic model for different context information, and therefore context information can be easily expressed, reasoned and shared. Currently, there are many context modeling modes. Various modeling modes have own advantages and disadvantages with different focuses. The first RFID information model is proposed by Harrison and other researchers in the literature [5] [6]. He takes the form of events for RFID information modeling. State history of object and temporal semantics of business flow are implicit. Support can not be effectively provided for complex inquiry, such as inquiry during object tracking and monitoring. A query usually should be completed in many steps indirectly and inefficiently. Wang Licai, Meng Xiangwu, etc. summed up current context modeling method, and they pointed out 
that model-based method has the problem of difficult calculation of high-dimensional data similarity [7]. One prediction model should be constructed or updated by longer time aiming at model-based method. A lot of parameters should be adjusted for optimization. When user preference data is very small, the data may not be enough to build reliable classification. Since method based on support vector machine (SVM) itself has the defects that large-scale training sample can not be easily handled and 'various classification problems can not be solved easily', etc., it is limited in application of context modeling.

\section{Context Modeling in Mobile Learning}

Mobile learning system model based on RFID is constructed here. RFID-based mobile learning system is designed and realized. The system can be applied in schools, libraries, museums, zoos, historical sites, botanical garden and other real life environment. Learners can produce close situation interaction with real environment in the learning process through the system. Learners can obtain desired learning information without manually inputting learning information demand, thereby improving learning interests and learning efficiency of learners, and the application scene is shown in Fig.1.

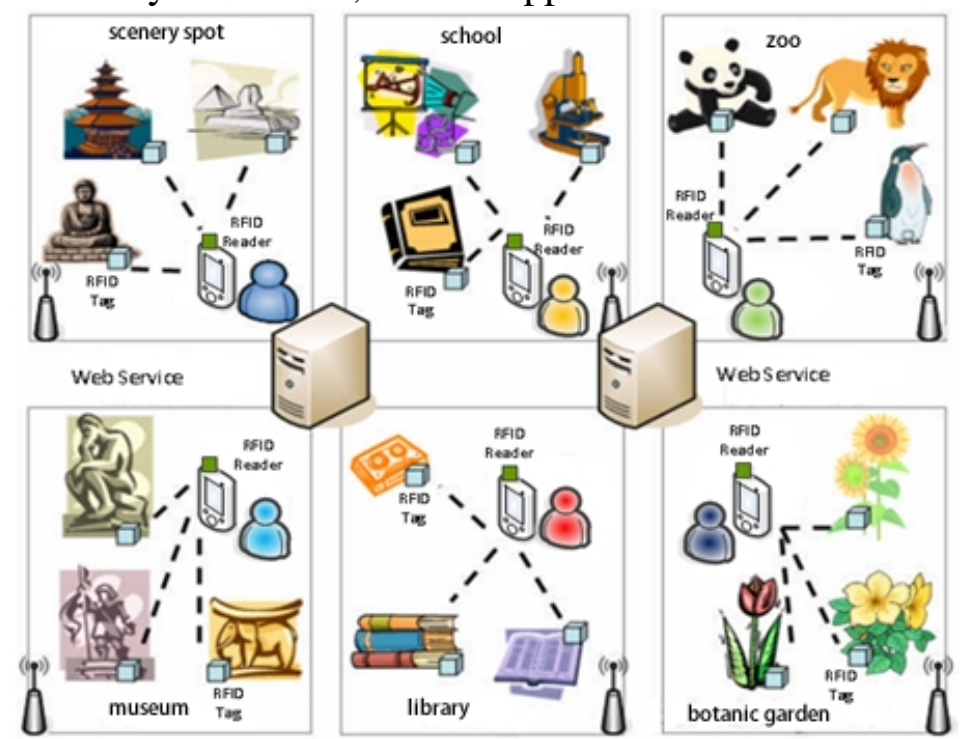

Fig. 1 RFID-based mobile learning system application scene

In the system, RFID Tag is pasted on the learning object, and related learning resources are connected through RFID Tag. Learners can hold mobile terminal with RFID Reader by hand (such as PDA) for reading Tag tags attached on learning object, learning object can interact with mobile terminal for displaying corresponding learning information. Therefore, learners can stay in the mobile learning stably, and original learning effect can not be affected due to obtaining unsuitable learning information. In addition, learners also can learn according to personal interest.

Learning model is shown in Fig.2. Learning objects attached with RFID Tag belongs to the perceived layer. Mobile terminals with RFID read-write device held by learners belong to perceiving layer, and Web server and database server belong to service layer.

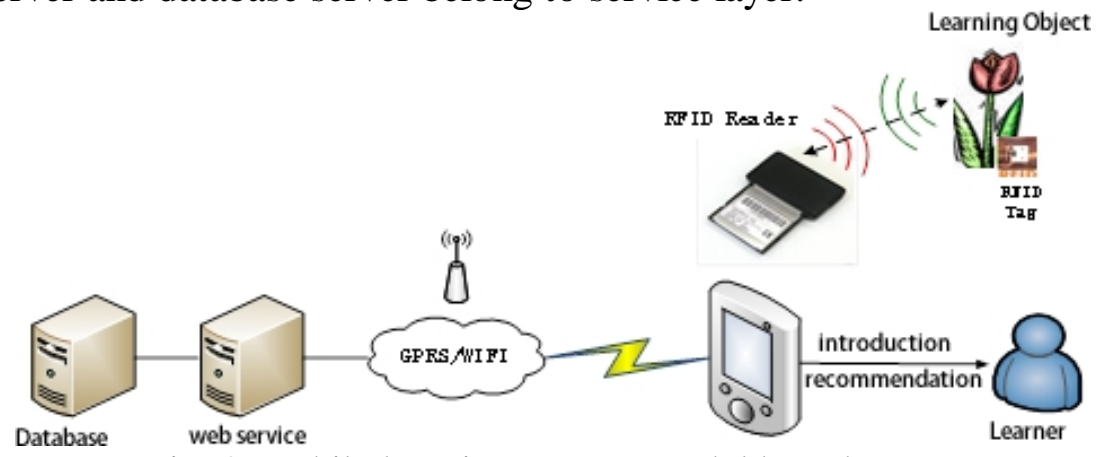

Fig. 2 Mobile learning system model based on RFID 
The learner's learning process is shown as follows:

a) When learners use mobile terminals with RFID read-write device for reading RFID Tag tags of learning objects, RFID Tag is activated by RFID read-write device of mobile terminal;

b) Identification information (such as ID) in RFID Tag is transmitted to RFID read-write device by radio frequency. The identification ID information of learning objects is parsed out through treatment;

c) Mobile terminal visits Web servers or Web services server through using parsed ID by GPRS or WIFI and other wireless network on the basis of HTTP protocol or SOAP message;

d) Learning information can be sent to mobile terminal for learners to learn through wireless network by treatment with server.

In the process of learning, learners' learning activities will be recorded to the backend database, reference can be provided for the system to recommend suitable learning information to the learners. After learners learn for certain time with the mobile client, the system can recommend some suitable learning information to the learners according to the learners' learning ability, learning record and IRT theory. Learners can select own interested content for learning from the learning information recommended by the system.

\section{Learning Recommendation Algorithm Based on Context Awareness}

Recommendation learning refers that learning content consistent with learners' ability is recommended according to previous learning condition of the learners. Maximum likelihood estimation method is firstly adopted for assessing ability of learners in order to recommend next learning information. Learning information database and examination question information database meeting four basic assumptions of examination question response theory are established. On the basis, we divide learners' ability into seven levels from -3 to +3 according to traditional classification method. Eq.1- maximum likelihood estimation method is utilized for assessing learners' ability according to learning information condition of learners. The maximum information function is regarded as recommendation information. Eq.4 shows that the learning information and examination question information difficulties are divided into five levels from -2 to +2 . Learning information with corresponding difficulty can be searched back in the database as recommendation according to the learning information difficulty obtained by maximum difficulty information function [8].

$$
L\left(r_{1}, r_{2}, \ldots, r_{n} \mid \theta_{k}\right)=\prod_{i=1}^{n} P_{i}\left(\theta_{k}\right)^{r_{i}} Q_{i}\left(\theta_{k}\right)^{1-r_{i}}
$$

Parameter explanation:

$\theta_{k}$ represents learner's ability;

$r_{1}, r_{2}, r_{3} \ldots r_{n}$ represents the response condition of learners to the examination question information, 1 for correct answer, and 0 for wrong answer on the contrary.

$P_{i}\left(\theta_{k}\right)$ represents the probability of the learners to answer the ith question under $\theta$ question. $Q_{i}\left(\theta_{k}\right)$ represents the probability of wrongly answering the ith question.

We can use Eq.2 to calculate the probability of the learners to correctly answering the ith question information:

$$
P_{i}(\theta)=C_{i}+\left(1-C_{i}\right) \frac{e^{D a_{i}\left(\theta-b_{i}\right)}}{1+e^{D a_{i}\left(\theta-b_{i}\right)}}
$$

Wherein, $\mathrm{i}=1,2, \ldots, \mathrm{n}, \mathrm{D}=1.7$,

$\theta$ : it represents learners' ability value, and we represent ability value of the learner with -3 to +3 .

$P_{i}(\theta)$ : it represents the probability of learners with ability $\theta$ to correctly answer the ith question information;

$a_{i}$ : it represents discrimination parameter of the ith question;

$b_{i}$ : it represents the difficulty parameter of the ith question; 
$C_{i}:$ it represents the guess parameter of the ith question;

Constant $\mathrm{D}$ aims at making examination question response mode close to Normal Ogive Model. $\mathrm{D}=$ 1.7.

In the system, we ignore guess parameters and discrimaination parameter, and Eq. 3 is utilized for representing the probability of learners with ability $\theta$ in correctly answering the ith examination question. The result $P_{i}(\theta)$ represents correct answer. Similarly, $1-P_{i}(\theta)$ represents the probability of wrong answer, which respectively correspond to $P_{i}\left(\theta_{k}\right)$ and $Q_{i}\left(\theta_{k}\right)$ in Eq.1.

$$
P_{i}(\theta)=\frac{e^{D\left(\theta-b_{i}\right)}}{1+e^{D\left(\theta-b_{i}\right)}}
$$

Since ability value parameters are between -3 and +3 , we convert the result into the scope according to percentage, and then it is taken into Eq.1, thereby difficulty of recommended information can be calculated according to comprehension.

Learners' ability is usually divided from -3 to +3 according to constant $\mathrm{D}$. The question difficulty is from -2 to +2 . If the learner's ability is -3 , it represents that the learner has poor ability. If the learner's ability is 0 , it represents that the learner has medium ability. If the learner's ability is +3 , it represents that the learner has good learning ability. Representation methods of examination question difficulty are the same as ability of learners, which are classified from low level to high level.

We consult Eq.4 according to the ability value information for obtaining the biggest difficulty information. Suitable learning information is recommended according to difficulty information value, thereby facilitating improvement of learners.

$$
I_{j}(\theta)=\frac{(1.7)^{2}}{\left[e^{1.7\left(\theta-b_{j}\right)}\right]\left[1+e^{-1.7\left(\theta-b_{j}\right)}\right]^{2}}
$$

Learning ability of learners is firstly inquired in learning information recommendation services in the aspect of concrete implementation. Learning information can be recommended to learner by two different modes according to whether the learning ability is empty or not: (1) If the learning ability of the learner is empty, the learning information of the learning objects with the highest learning frequency in the learning record can be recommended to the learner; (2) if the learners' learning ability is not empty, learning information with difficulty matched with the learner's learning ability can be recommended to the learner according to Eq.4 the largest difficulty information function. Then, the recommended learning information list can be returned to mobile client by learning information recommendation services. Finally, client procedures can display the learning information list returned by the server on the mobile equipment for learners to select. The flowchart is shown in Fig. 4.

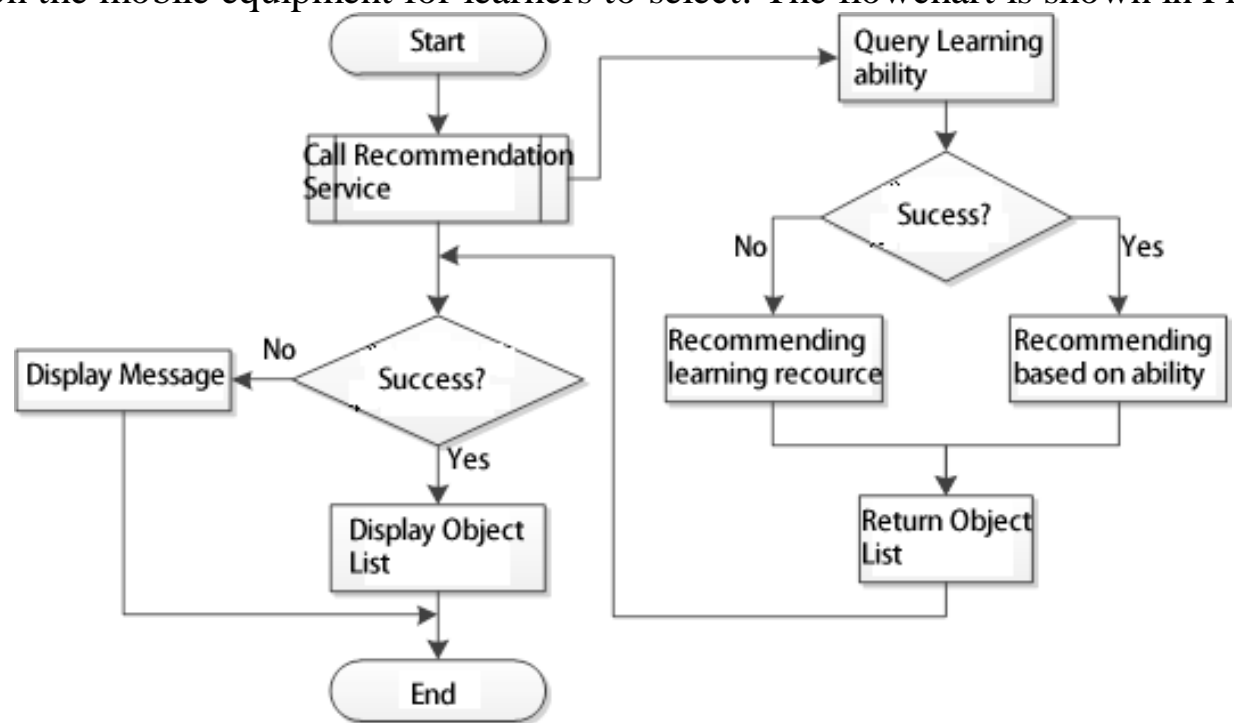

Fig. 3 Learning information recommendation module flowchart 


\section{Conclusions}

After context information characteristics in the application are analyzed, characteristics of context model aiming at RFID application are determined. Context model aiming at object is utilized for realizing context information modeling in the RFID application from the aspects of expressiveness, verifiability, extensibility, memory and query efficiency. The established model is compatible to existing RFID data model specification. Structured representation mode is adopted for representation. Entity relationship and temporal information can be clearly expressed. Information validation and expansion are supported. Quick memory and inquiry of context information can be realized. It is very complicated to design and realize mobile learning system. Many problems should be further studied and discussed deeply, such as difference between simulator and actual mobile devices, different mobile device and different network access modes can affect use effect of the users. The problems should be correspondingly improved aiming at the system in subsequent study.

\section{Acknowledgement}

This work was supported by a grant from the Science \& Research Plan Project of Shaanxi Province Department of Education (12JK0737) .

\section{References}

[1] R. Mugwanya and G. Marsden. Mobile learning content authoring tools (MLCATs): A systematic review. Proc. 1, vol. 38, pp. 20-31, 2010

[2] T. Heimonen, M. Turunen, S. Kangas, Seek'N'Share: A platform for location-based collaborative mobile learning. Proc. 12th Int. Conf. Mobile Ubiquitous Multimedia, pp. 1-4, 2013

[3] Wenqian Xiong. Design and Implementation of Mobile Learning System Based on Windows Mobile Platform [D].Beijing: Beijing University of Post and Telecommunications, 2010.

[4] N. J. Ahuja and R. Sille. A critical review of development of intelligent tutoring systems: Retrospect, present and prospect. Int. J. Comput. Sci. Issues, vol. 10, no. 4, pp. 39-48, 2013

[5] Harrison, M. EPC information service - Data model and queries. Technical Report,Auto-ID center, 2003.

[6]Harrison, M., Brusey, J., Moran, H., McFarlane, D. PML server developments. Technical Report, Auto-ID center, October 2003.

[7] C. Marinagi, C. Skourlas and P. Belsis. Employing ubiquitous computing devices and technologies in the higher education classroom of the future. Procedia-Social Behavioral Sci., vol. 73, pp. 487-494, Feb. 27, 2013

[8] S. Gómez, P. Zervas, D. G.Sampson and R. Fabregat. Context-aware adaptive and personalized mobile learning delivery supported by UoLmP. J. King Saud University-Comput. Inform. Sci., vol. 26, no. 1, pp. 47-61, Jan., 2014 